\title{
GSK-3 $\alpha$ is a central regulator of age-related pathologies in mice
}

\author{
Jibin Zhou, ${ }^{1}$ Theresa A. Freeman, ${ }^{2}$ Firdos Ahmad, ${ }^{1}$ Xiying Shang, ${ }^{1}$ Emily Mangano, ${ }^{3}$ \\ Erhe Gao, ${ }^{1}$ John Farber, ${ }^{4}$ Yajing Wang, ${ }^{5}$ Xin-Liang Ma, ${ }^{5}$ James Woodgett, ${ }^{6}$ \\ Ronald J. Vagnozzi, ${ }^{1,4}$ Hind Lal, ${ }^{1}$ and Thomas Force ${ }^{1}$
}

\begin{abstract}
${ }^{1}$ Center for Translational Medicine, Temple University School of Medicine, Philadelphia, Pennsylvania, USA. 2Department of Orthopaedic Surgery, Thomas Jefferson University, Philadelphia, Pennsylvania, USA. ${ }^{3}$ Brigham and Women's Hospital and Harvard Medical School, Boston, Massachusetts, USA. ${ }^{4}$ Department of Pathology, Anatomy and Cell Biology and ${ }^{5}$ Department of Emergency Medicine, Thomas Jefferson University, Philadelphia, Pennsylvania, USA. ${ }^{6}$ Samuel Lunenfeld Research Institute, Mount Sinai Hospital, Toronto, Ontario, Canada.
\end{abstract}

\begin{abstract}
Aging is regulated by conserved signaling pathways. The glycogen synthase kinase-3 (GSK-3) family of serine/ threonine kinases regulates several of these pathways, but the role of GSK-3 in aging is unknown. Herein, we demonstrate premature death and acceleration of age-related pathologies in the Gsk3a global KO mouse. KO mice developed cardiac hypertrophy and contractile dysfunction as well as sarcomere disruption and striking sarcopenia in cardiac and skeletal muscle, a classical finding in aging. We also observed severe vacuolar degeneration of myofibers and large tubular aggregates in skeletal muscle, consistent with impaired clearance of insoluble cellular debris. Other organ systems, including gut, liver, and the skeletal system, also demonstrated age-related pathologies. Mechanistically, we found marked activation of $\mathrm{mTORC1}$ and associated suppression of autophagy markers in KO mice. Loss of GSK-3 $\alpha$, either by pharmacologic inhibition or Gsk3a gene deletion, suppressed autophagy in fibroblasts. mTOR inhibition rescued this effect and reversed the established pathologies in the striated muscle of the KO mouse. Thus, GSK-3 $\alpha$ is a critical regulator of mTORC1, autophagy, and aging. In its absence, aging/senescence is accelerated in multiple tissues. Strategies to maintain GSK-3 $\alpha$ activity and/or inhibit mTOR in the elderly could retard the appearance of age-related pathologies.
\end{abstract}

\section{Introduction}

Aging is usually defined as the progressive loss of function accompanied by decreasing fertility and increasing mortality with advancing age (1). It is a complex biological process controlled by multiple genetic, epigenetic, and environmental factors. In order to explain how aging occurs at the molecular level, numerous theories have been proposed, but as yet, a unifying theory has not emerged. There are four main theories that are accepted more widely. (a) The telomere loss theory proposes that telomere shortening represents a cell-intrinsic mechanism, leading to DNA damage accumulation and activation of DNA damage checkpoints in aging cells. Activation of DNA damage checkpoints in response to telomere dysfunction results in induction of cellular senescence (2-4). (b) The somatic mutation theory states that aging proceeds if somatic mutations and other forms of DNA damage exceed the capacity for DNA repair (5). (c) The mitochondrial theory suggests that accumulation of mutations in mitochondrial DNA with age impairs ATP production, resulting in impaired bioenergetics (4). (d) The waste accumulation theory proposes that aging results from the accumulation of damaged proteins or superfluous or dysfunctional organelles due to age-related impairment of degradative processes, including the ubiquitin-proteasome system and, especially, lysosome-mediated autophagy $(6,7)$.

Many conserved signaling pathways and regulatory proteins are reported to regulate life span and rate of aging of eukaryotic organisms. They include, but are not limited to, the insulin/IGF-1 pathway, the mTOR pathway, the WNT signaling pathway, and the $\mathrm{p} 53 /$ sestrin signaling pathway. The insulin/IGF-1 signaling cascade comprises insulin/IGF-1, insulin/IGF-1 receptor/DAF-2,

Conflict of interest: The authors have declared that no conflict of interest exists. Citation for this article: J Clin Invest. 2013;123(4):1821-1832. doi:10.1172/JCI64398.
insulin/IGF-1 receptor substrate (IRS), phosphatidylinositol 3-kinase, 3-phosphoinositide-dependent protein kinase-1, AKT/ $\mathrm{PKB}$, and the FOXO/DAF-16 transcription factor $(8,9)$. Multiple mutations in components of this signaling pathway extend life span, e.g., mutations in DAF-2 or IRS double the life span of C. elegans (10). This extension of life span is also seen in heterozygous IGF-1 KO mice (null mutants are not viable) (11) and in mice lacking the insulin receptor in adipose tissue (12).

The mTOR kinase is a key amino acid and nutrient sensor that stimulates growth and blocks salvage pathways, such as autophagy, when energy stores are plentiful (13). mTOR exerts its effects by phosphorylating eukaryotic initiation factor 4Ebinding (eIF4E-binding) protein 1 (4E-BP1), which inhibits 5 '-cap-dependent mRNA translation (the major mechanism of translation) by binding and inactivating eIF4E. Phosphorylation of 4E-BP1 leads to release of eIF4E, allowing initiation of translation (14). In addition to $4 \mathrm{E}-\mathrm{BP} 1, \mathrm{mTOR}$ also regulates translation via 66 kinase. Inhibiting the mTOR pathway increases life span in many species, from yeast to mice $(15,16)$. Increased WNT signaling was recently reported to be a potent activator of mitochondrial biogenesis and ROS generation, leading to DNA damage and acceleration of cellular senescence in primary cells (17). p53 is a well-established transcription factor, with tumorsuppressive properties (18). Sestrins, which are target genes of $\mathrm{p} 53$, have been reported to protect cells against various insults through functioning as antioxidants, thereby reducing ROS accumulation. Sestrins also act as inhibitors of TORC1 signaling, preventing accelerated aging and development of age-associated pathologies (19-21). Klotho has been identified as an "aging suppressor" in mice (22). Deletion of klotho appears to lead to accelerated aging in mice, due, in part, to augmented WNT signaling (23). 


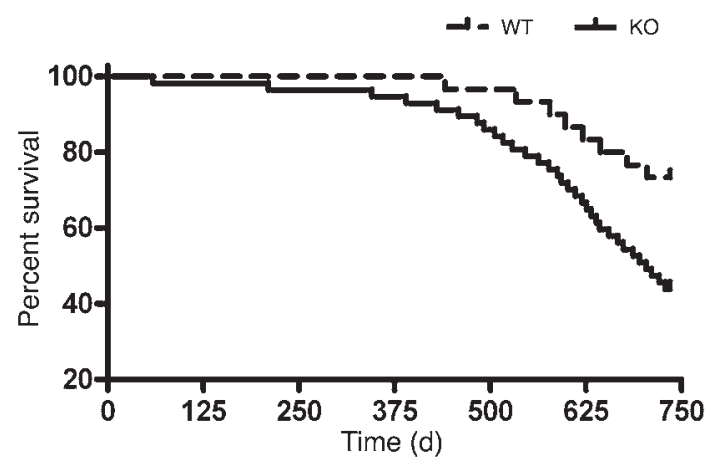

Figure 1

Survival analysis in Gsk3a KO and WT mice. Cumulative survival curves in $n=57$ Gsk3a KO and $n=30$ WT mice. Kaplan-Meier survival analysis confirms shortened life span in the Gsk3a KO mouse. The percentage of survival at termination of the study (24 months) was $42 \%$ for Gsk3a KO mice and $73 \%$ for WT mice $(P=0.01)$.

The glycogen synthase kinase-3 (GSK-3) family of serine/threonine kinases was first identified as a negative regulator of glycogen synthase, the rate-limiting enzyme in glycogen synthesis (24). The family consists of 2 isoforms, $\alpha$ and $\beta$, which are $98 \%$ identical within their kinase domains but differ substantially in their Nand C-terminal sequences. Unlike most protein kinases, GSK-3 is typically active in unstimulated cells and is inhibited in response to a variety of inputs (25). Because GSK-3-mediated phosphorylation of substrates usually leads to inhibition of those substrates, the net result of inhibition of GSK-3 is typically functional activation of its downstream substrates.

Few enzymes exert as broad a regulatory influence on cellular function as GSK-3. Over 50 targets have been reported to be phosphorylated by GSK-3, including metabolic enzymes, signaling molecules, structural proteins, and transcription factors. Thus, it is not surprising that GSK-3 plays important roles in numerous signaling pathways that regulate a variety of cellular processes (26-30). Importantly, we noted that a number of the factors discussed above that regulate aging have been reported to be regulated by GSK-3s, including the WNT, insulin/IGF-1, mTOR, and p53 signaling pathways. Herein, we present what we believe to be the first studies demonstrating accelerated development of aging-related pathologies in striated muscle (heart and skeletal muscle - our primary focus) but also in gut, liver, and joints (bone and cartilage) in a Gsk3a KO mouse. These phenotypes are associated with a reduced life span. We believe that the evidence suggests that GSK-3 $\alpha$ is a novel regulator of aging that retards age-related pathologies in a wide variety of tissues. Our studies, including those with everolimus, an mTOR inhibitor, implicate unrestrained mTOR activity as a key factor driving aging in the absence of GSK-3 $\alpha$ and suggest that mTOR-mediated impairment of autophagy is the critical downstream event promoting senescence.

\section{Results}

Shortened life span in the Gsk3a KO mouse. We chose to focus on GSK-3 $\alpha$ largely because of a chance observation that Gsk3a KO mice appeared to die earlier than WT littermates. To determine whether this was the case, we applied Kaplan-Meier analysis to a cohort of mice. We followed $57 \mathrm{KO}$ and $30 \mathrm{WT}$ age-matched mice, with daily observation for deaths. A survival disadvantage in the $\mathrm{KO}$ mice first became statistically significant at 534 days ( 76.3 weeks) of age $(P=0.04$; Figure 1$)$. The percentage of survival at termination of the study (24 months or 104 weeks) was $42.1 \%$ for the KO mice and $73.3 \%$ for WT mice $(P=0.011)$. While it is difficult to pinpoint the exact cause of death, due to the marked alterations in a number of organ systems, given the very profound cardiac abnormalities observed in the KO mice (see below), we presume the vast majority of deaths were cardiac in origin.

Cardiac bypertrophy, contractile dysfunction, impaired diastolic relaxation, and senescence in the Gsk3a KO mice. We then examined the hearts of the Gsk3a KO mice. We had previously reported that this mouse developed spontaneous cardiac hypertrophy, beginning after 6 months of age (31). In order to extend the time line, we studied $\mathrm{KO}$ and littermate control mice at 3, 6, 12, and 24 months. Of note, we observed no alteration in phosphorylation status or total levels of GSK-3 $\alpha$ in WT mice across this age range (Supplemental Figure 1; supplemental material available online with this article; doi:10.1172/JCI64398DS1). We first confirmed that the KO mice had more hypertrophy at 6 months, but this continued to worsen over time, whether based on direct quantification of heart weight or echocardiographic determination (Figure 2, A-D). More strikingly, contractile dysfunction and diastolic relaxation, as determined by invasive hemodynamic monitoring, were dramatically worse in the KO mice (Figure 2, E-G). Studies using echocardiography also showed impaired contractile function, with significant reductions in ejection fraction (EF) (Figure 2H). Furthermore, dilative remodeling was pronounced, with marked increases in the size of the LV chamber (Figure 2I).

We then examined the myocardia of the $\mathrm{KO}$ mice at the various ages. $\mathrm{H} \& \mathrm{E}$ staining of the heart revealed vacuolar degeneration and blanching of the myocardium, consistent with marked sarcopenia, a hallmark of aging in muscle (Figure 3A). This was evident as early as 12 months of age. In other sections, we saw disappearance of sarcomeric structures and loss of myofibrils in the $\mathrm{KO}$ mice but not in the age-matched WT mice (Figure 3C). We also saw marked myocyte dropout with increasing fibrosis on both H\&Eand trichrome-stained sections (Figure 3, B and C). Using transmission electron microscopy, we saw large numbers of swollen and structurally disrupted mitochondria (Figure 3D). Consistent with this, there were increased levels of ROS in the KO mice, as determined by superoxide production (Figure 3E). Finally, expression of p16, a marker of senescence, was significantly increased in the hearts of the $\mathrm{KO}$ mice (Figure 3F).

Skeletal muscle sarcopenia and tubular aggregates in the KO mouse. Given the findings in the heart, we next examined skeletal muscle in the $\mathrm{KO}$ mouse. In the vastus intermedius, we observed vacuolar degeneration similar to that seen in the heart (Figure 4A). This was not present at any age in the WT controls. In addition, we observed tubular aggregates in the $\mathrm{KO}$ mice that again were not present in WT mice at any age (Figure 4B). Tubular aggregates are cytoplasmic organelles containing miscellaneous proteins, including proteins of the sarcoplasmic reticulum and mitochondria. They are insoluble and can be transferred to vacuoles but are also an alternate system to traffic transport-incompetent insoluble material. They appear to become more important when more traditional rescue systems (e.g., autophagy) are impaired. In addition, they are purported to exacerbate myopathies in some situations $(32,33)$. As with the heart, superoxide production was significantly increased in the skeletal muscle (Figure 4C). 
A

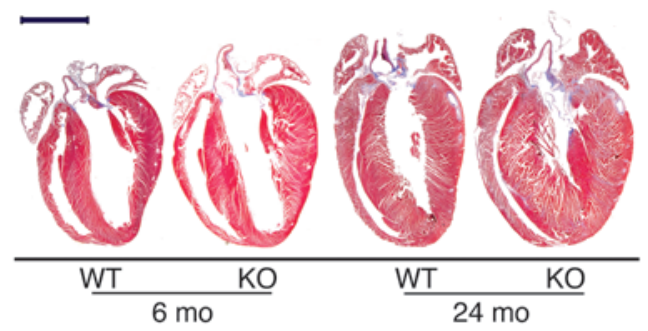

B

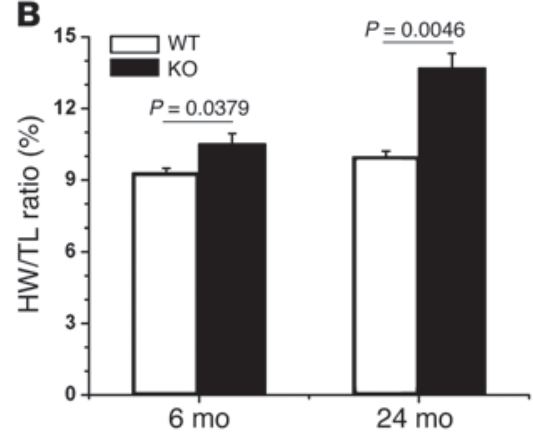

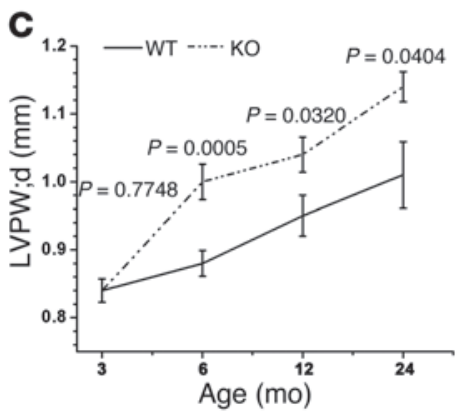

D

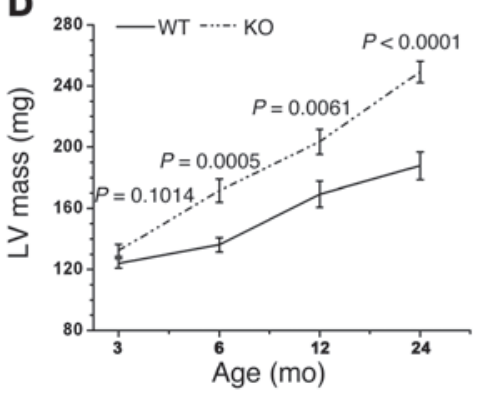

E

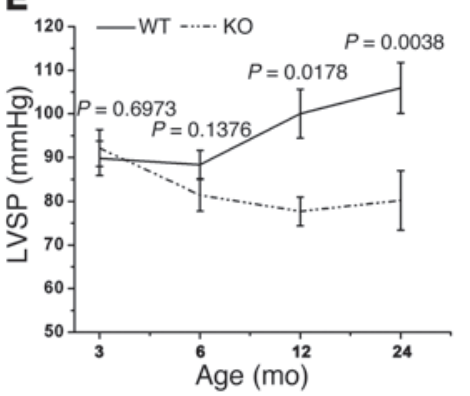

$\mathbf{F}$
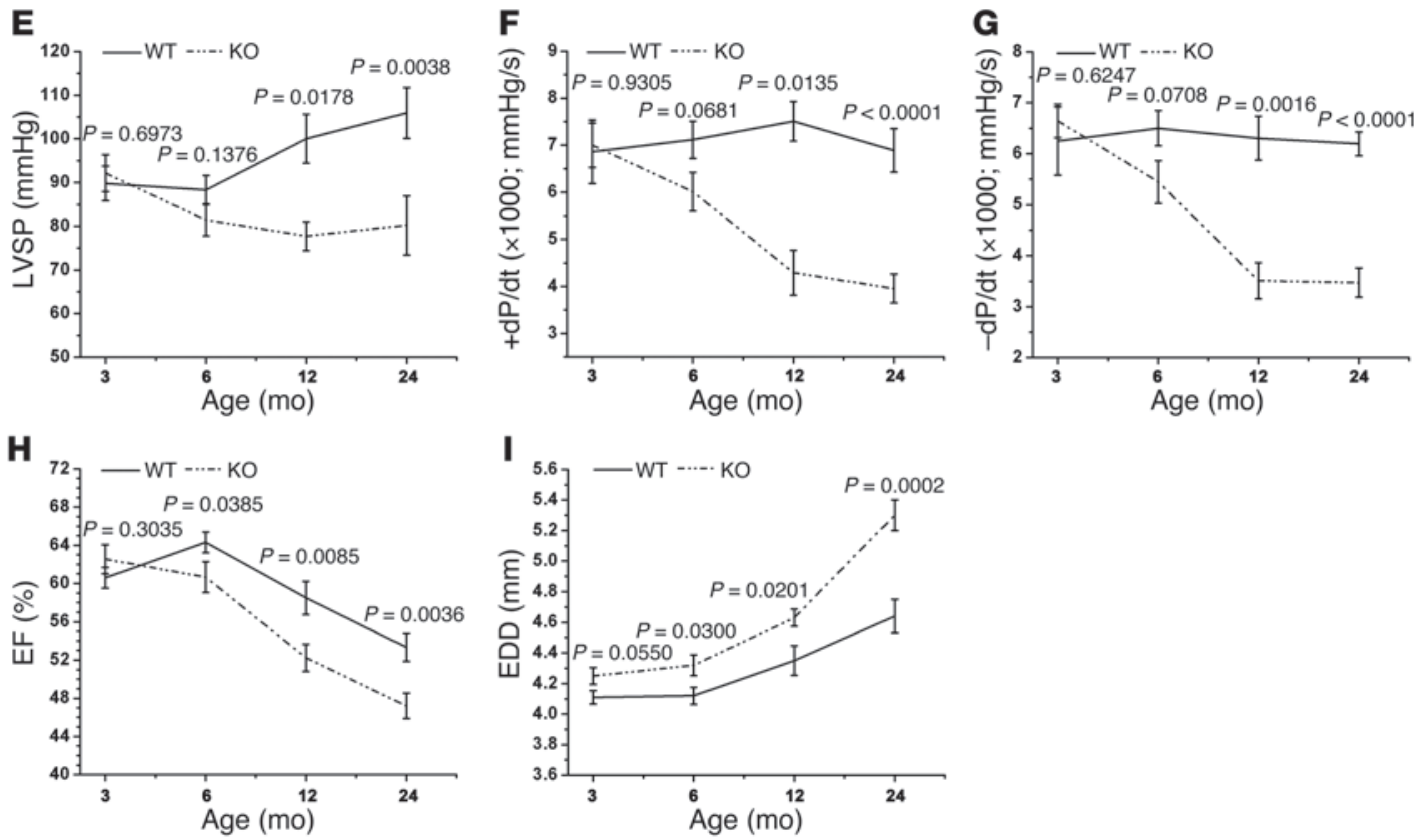

\section{Figure 2}

Cardiac abnormalities in the Gsk3a KO mouse. Deletion of GSK-3 $\alpha$ leads to exaggerated cardiac hypertrophy, contractile dysfunction, and impaired diastolic relaxation. Mice were examined for cardiac abnormalities at (A and B) 6 months and 24 months of age or (C-I) at 3, 6, 12, and 24 months. (A) Representative images of hearts of KO and WT mice. Original magnification, $\times 2$; scale bar: 4 mm. (B) Quantification of hypertrophy using heart weight normalized to tibial length (HW/TL). Hypertrophy as determined by $(\mathbf{C})$ transthoracic echocardiographic assessment of LV posterior wall thickness or by (D) direct measurement of LV mass. (E-G) Hemodynamic parameters determined at cardiac catheterization: (E) LV systolic pressure (LVSP), (F) $+\mathrm{dP} / \mathrm{dt}$ (a measure of contractile function), and (G) $-\mathrm{dP} / \mathrm{dt}$ (a measure of the ability of the heart to relax) are all impaired in the KO mouse. (H) Contractile function, as assessed by echocardiography, is impaired. (I) EDD, a marker of dilatation of the LV, is increased in the KO mouse.

Senescence in other organ systems. Although our focus was on striated muscle, we also wanted to determine whether senescence might affect other organ systems in the KO mouse. Therefore, we turned to the digestive system and examined the liver and small intestine. Surprisingly, given what we had seen in other organ sys- tems, deletion in the liver caused no obvious abnormalities on $\mathrm{H} \& \mathrm{E}$ staining (data not shown). However, when we looked for markers of senescence in the livers of the $\mathrm{KO}$ mice, we found a highly significant increase in phospho-histone H2AX-positive cells, consistent with premature senescence in $\mathrm{KO}$ hepatocytes (Figure 5A). 
A

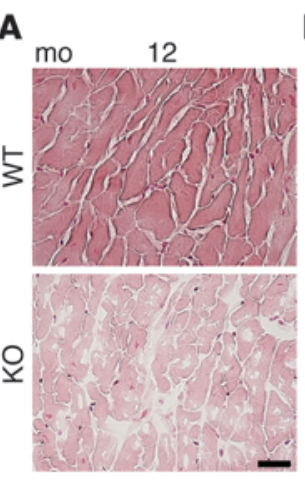

B

C
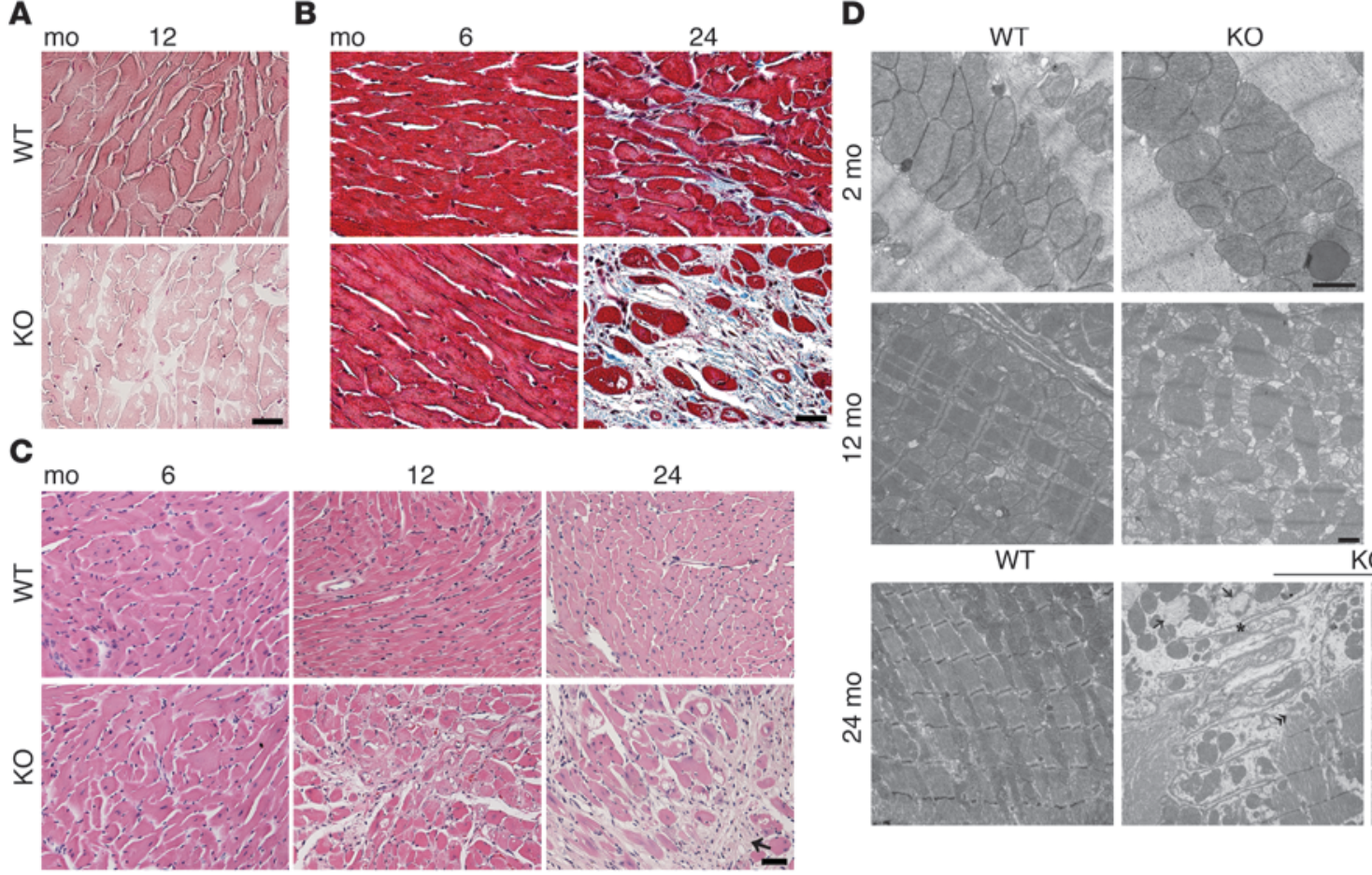

24

E
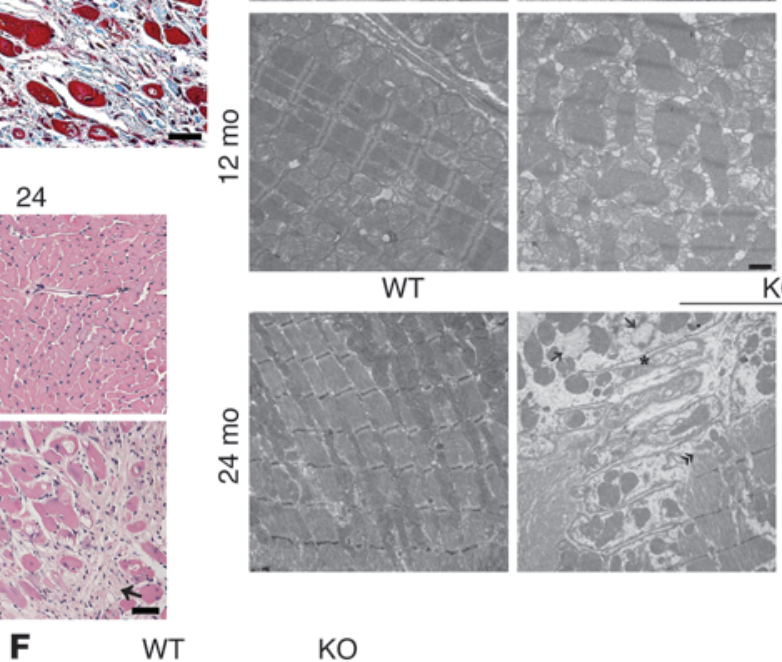

WT
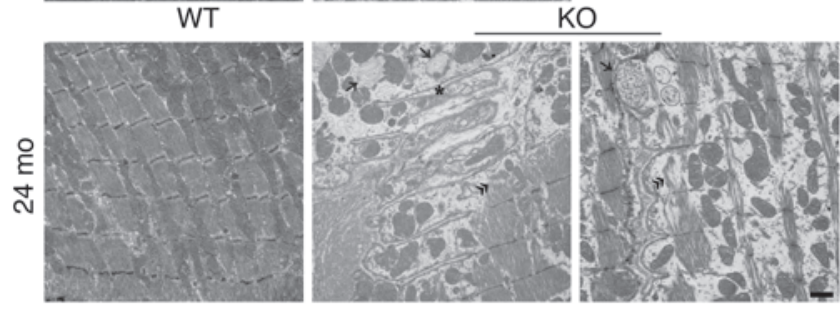

$\mathrm{KO}$
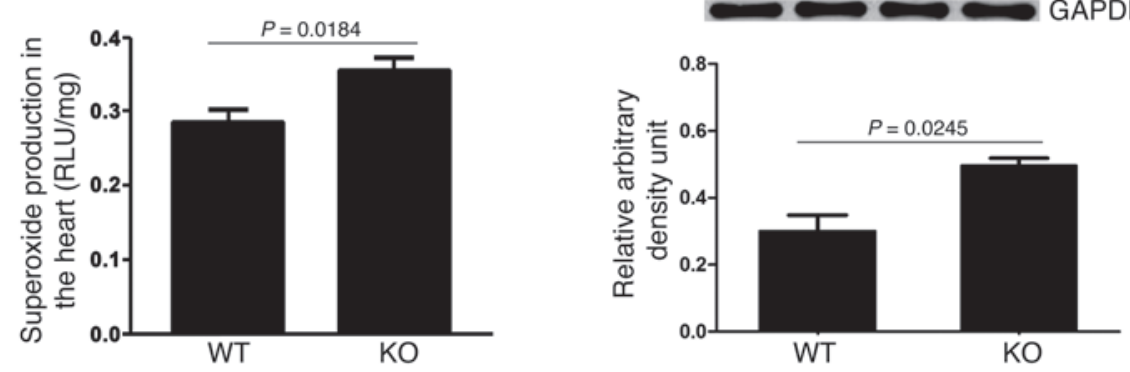

\section{Figure 3}

Sarcopenia, myocyte dropout, and progressive fibrotic remodeling in the heart of the KO mouse. (A) Vacuolar degeneration and blanching of the myocardia, consistent with marked sarcopenia and myocyte dropout, are apparent in the H\&E-stained hearts of KO mice. Original magnification, $\times 400$; scale bar: $20 \mu \mathrm{m}$. (B) Fibrotic remodeling is shown in the Masson's trichrome-stained sections. Original magnification, $\times 400$; scale bar: $20 \mu \mathrm{m}$. (C) Additional H\&E-stained images show myocyte dropout and fibrosis that is readily apparent as early as 12 months of age in the KO mice. The arrow indicates area of myocyte dropout. Original magnification, $\times 200$; scale bar: $100 \mu \mathrm{m}$. (D) Representative transmission electron micrographs in the heart at 2, 12, and 24 months of age. There are markedly swollen mitochondria, with marked disruption of sarcomeres, in the $\mathrm{KO}$ mice at 12 months of age, worsening at 24 months, at which point there is loss of mitochondria and myofibrils. Original magnification, $\times 10,000$ (top row); $\times 5,000$ (middle row); $\times 4,400$ (bottom row); scale bar: $1 \mu \mathrm{m}$. (E) Increased superoxide production in KO mice. (F) Increased p16 expression in the hearts of KO mice.

For the small intestine of the KO mouse, we used a marker of cellular senescence, senescence-associated $\beta$-galactosidase (SA $\beta$-gal) activity, and found a marked increase in activity in the KO mice, whereas only sporadic SA $\beta$-gal-positive single cells were seen in the WT mice (Figure 5B).

Bone and skeletal system. We next examined the skeletal system and joints. We used micro-CT and histological sections stained with $\mathrm{H} \& \mathrm{E}$ and Alcian blue (the latter to detect proteoglycan) to examine the knee joint for signs of age-related osteoarthritis. At 1 year of age bone volume/total volume (BV/TV) in the KO mice was similar to that in the WT mice (Figure 6A). Furthermore, the joints of WT and $\mathrm{KO}$ mice were comparable in architecture, and the bones and articular cartilage surfaces appeared relatively normal (Figure 6A). However, at 2 years of age, BV/TV in the $\mathrm{KO}$ mice was increased on micro-CT analysis (Figure 6A). This difference in bone volume between $\mathrm{KO}$ and WT mice can be clearly seen on the 3D reconstruction of the joint. The histological images confirm that the articular surface is denuded of cartilage, and ossification of the subchondral bone has extended into the meniscal area. The joint space of the $\mathrm{KO}$ mouse, as well as the surrounding support tissues, have both clearly mineralized, resulting in almost complete ankylosis.

To investigate the expression level of common mediators of osteoarthritis in these animals, immunohistochemistry, using antibodies to MMP-13 and IL-1, was performed (Figure 6B). The 
A

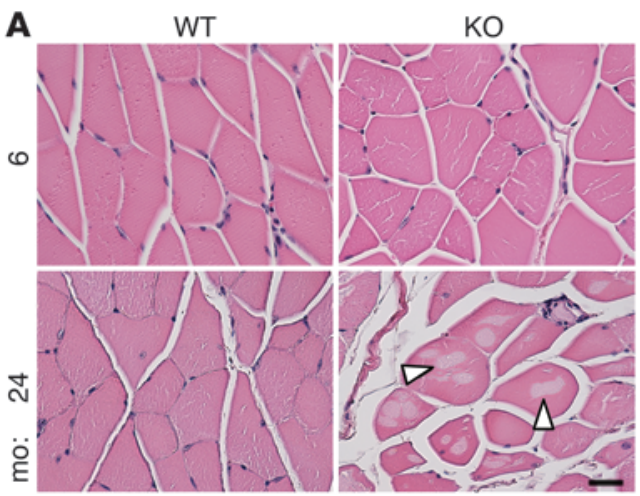

B
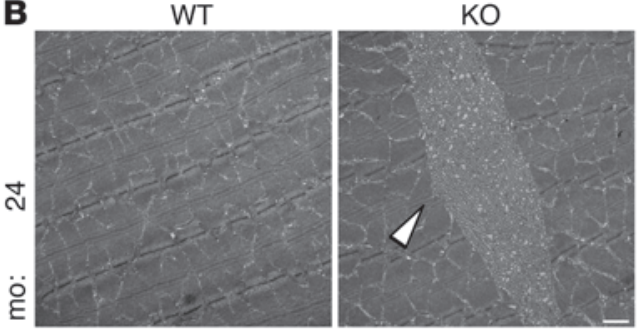

C

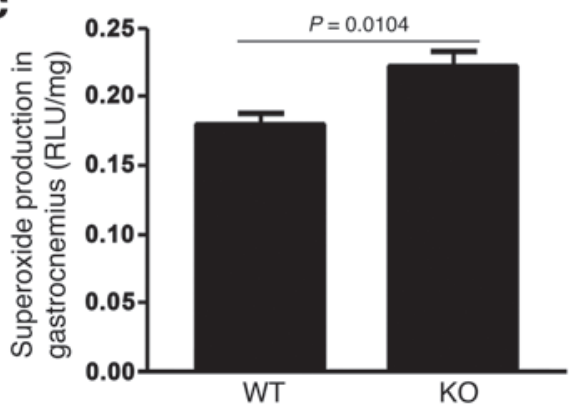

Figure 4

Vacuolar degeneration and tubular aggregates in the vastus intermedius of KO mice. (A) H\&E-stained sections showing vacuolar degeneration (arrows) of the myofibers at 24 months of age, which is not apparent in the WT mice. Original magnification, $\times 400$; scale bar: $50 \mu \mathrm{m}$. (B) Representative transmission electron micrograph showing a tubular aggregate (arrow) in the KO mouse. These were not seen in the WT mice. Original magnification, $\times 4,400$; scale bar: $1 \mu \mathrm{m}$. (C) Increased superoxide production in the skeletal muscle of the KO mice.

12-month-old mice showed similar amounts of MMP-13 at the osteochondral junction for both WT and KO animals. The main difference in MMP-13 staining between WT and KO mice was the higher level of expression by cells in the bone marrow of the KO mice. Similarly, the IL-1 staining at 1 year was also increased in the bone marrow, with additional distinct staining of osteocytes in the $\mathrm{KO}$ mice as compared with that in WT mice. At 24 months, the IL-1 staining appeared similar between WT and KO mice, but MMP-13 levels remained markedly increased in the $\mathrm{KO}$ mice. Thus, proinflammatory cytokines are indeed increased in the $\mathrm{KO}$ mice.

Mechanisms regulating aging. We next explored potential molecular mechanisms underlying the accelerated aging. We noted an increase in expression of IRS-1, a direct target of GSK-3, but, consistent with our prior studies (34), this was not associated with a significant increase in Akt activity, as determined by phospho-
A
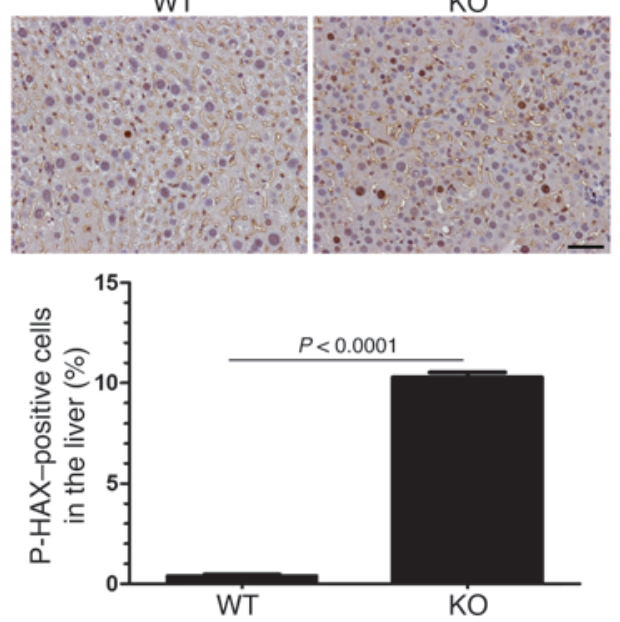

B

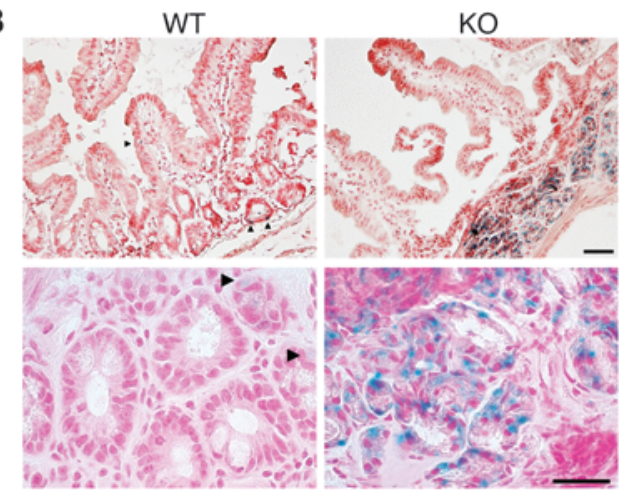

Figure 5

Senescence in the digestive system. (A) Senescence in the livers (hepatocytes) of the KO mice, as determined by phospho-histone H2AX positivity (brown-stained nuclei). Original magnification, $\times 200$; scale bar: $50 \mu \mathrm{m}$. (B) SA $\beta$-GAL, a marker of cellular senescence, is widespread in the small intestine of the KO mice (note blue areas). Although difficult to quantify, it appears that crypts are thin and villi are sparse in the KO mice, also consistent with senescence. Arrowheads indicate individual SA $\beta$-GAL-positive cells. Original magnification, $\times 100$ (top row); $\times 400$ (bottom row); scale bar: $200 \mu \mathrm{m}$ (top row), $50 \mu \mathrm{m}$ (bottom row). 
A mo:

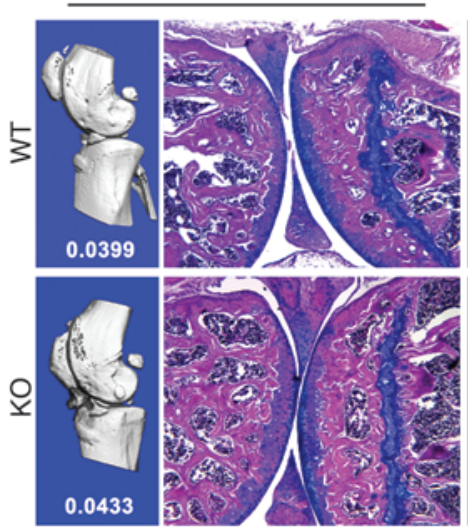

24

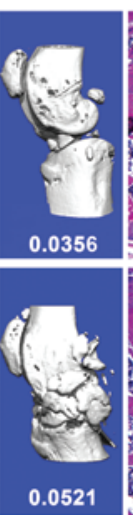

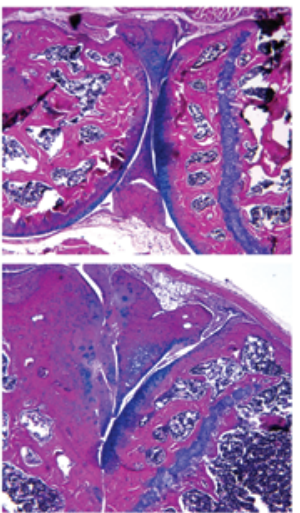

B mo: 12

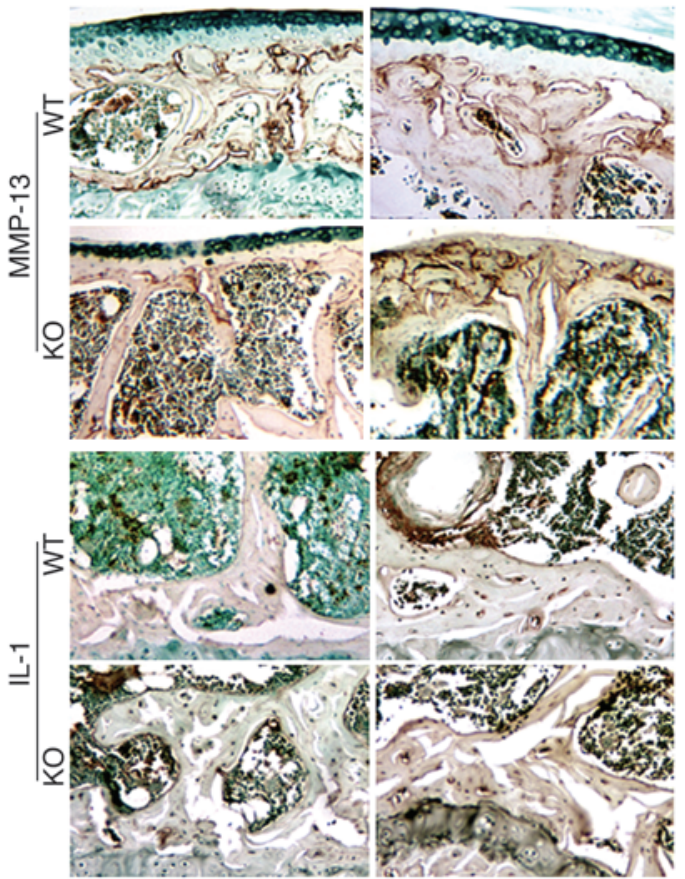

\section{Figure 6}

Increased bone mass, knee joint pathology, and inflammatory cytokines in the Gsk3a KO mice. (A) Representative micro-CT scans (in blue) and histological images of the knee. BV/TV, analyzed by micro-CT, was increased strikingly in the knees of the KO mice at 12 and 24 months of age. Values indicate BV/TV for each group. Calcification in the aging knees of the KO mice is also evident by both micro-CT and histology. There is fusion of the joint space and meniscus of the knee at 24 months of age. Original magnification, $\times 40$. (B) Representative immunohistochemistry images from the knees of WT or Gsk3a KO mice, stained for inflammatory cytokines MMP-13 and IL-1. MMP-13 staining (brown stain) is increased in the KO bone marrow at 12 months and remains elevated at 24 months. IL-1 staining (brown stain) is also increased in the osteocytes and bone marrow of the KO mice at 12 months, indicating elevated inflammatory cytokines in the KO mice. Original magnification, $\times 100$.

rylation of serine-473 of Akt (Figure 7, A and B). However, the dysregulation of mTORC1 function was most striking. We examined mTORC1 signaling in the aging mice and found markedly increased activity in the $\mathrm{KO}$ mice, based on phosphorylation of the 3 mTORC1 targets: 4E-BP1, S6 kinase, and ribosomal S6 protein (Figure 7, C-E). We also examined the phosphorylation status of tuberous sclerosis protein 2 (TSC2), which acts to inhibit mTOR. We found no alterations in TSC2 phosphorylation at T1462, a key Akt site (ref. 35 and Supplemental Figure 2), or at S1254, a site regulated by p38 MAPK (data not shown). This suggests that neither the Akt pathway nor p38 are major contributors to the increased mTORC1 activity seen in the KO mice.

Given the central role of $\mathrm{mTORC} 1$ in regulating autophagy and the key role of autophagy in aging, we assessed autophagy in the hearts of $\mathrm{KO}$ and WT mice by quantifying expression of the autophagy markers beclin-1 (ATG6), LC3-I/II, and p62. Beclin-1 expression was very evident in the hearts of the WT mice at 6 months of age but was largely reduced in the $\mathrm{KO}$ hearts, suggesting impaired or reduced autophagy (refs. 36, 37, and Figure 8A). Consistent with this, the LC3-II to LC3-I ratio was dramatically reduced in $\mathrm{KO}$ mice compared with that in WT mice, and this was particularly pronounced in the 12 - and 24-month-old mice (Figure 8A). Finally, p62 expression was markedly increased in the 12- and 24-month-old KO mice (Figure 8A). Thus, these 3 markers of autophagy are consistent with impaired autophagy in the $\mathrm{KO}$ mouse, particularly as it ages.
Despite these findings and the general support for using the above biomarkers of autophagy, it is accepted that autophagy should be measured as a "flux event" rather than a static measurement (38). Therefore, we used tandem mRFP-GFP-LC3 fluorescence analysis in mouse embryonic fibroblasts (MEFs) treated with a small molecule inhibitor of GSK-3 (SB216763; Figure 8, B and C, and ref. 39) and in Gsk3a KO adult fibroblasts (Figure 8, D and E) to determine whether GSK-3 $\alpha$ truly regulates autophagy. To summarize, both models were entirely consistent with GSK-3 $\alpha$ directly regulating autophagy. Inhibition of GSK-3 with the small molecule inhibitor significantly decreased autophagosome and autolysosome number and thus impaired autophagic flux (Figure 8B). SB216763 treatment also decreased the number of autophagosomes in the presence of bafilomycin A1, an inhibitor of autophagosome-lysosome fusion (Figure $8 \mathrm{C}$ ), suggesting that GSK-3 is also required for autophagosome formation. To further confirm the role of GSK-3 $\alpha$ in autophagic flux, tandem mRFP-GFP-LC3 assays were performed on isolated WT and Gsk3a KO adult fibroblasts. Starvation-induced autophagic flux was impaired in the Gsk3a KO fibroblasts (Figure 8, D and E, and Supplemental Figure 3). In these experiments, treatment with bafilomycin A1 significantly reduced autophagosome number in the Gsk3a KO fibroblasts compared with that in WT fibroblasts, confirming the role of GSK-3 $\alpha$ in autophagosome formation.

Finally, we wanted to determine the key driver(s) of the profound phenotypes that we observed in striated muscle of the Gsk3a KO mice, with our hypothesis being that unrestrained activation of 
A
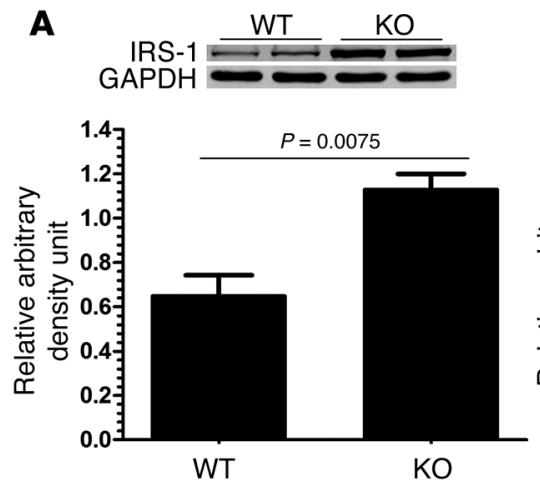

B
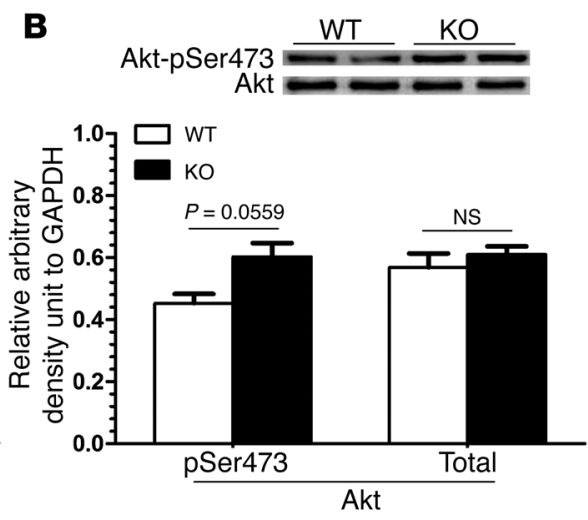

C
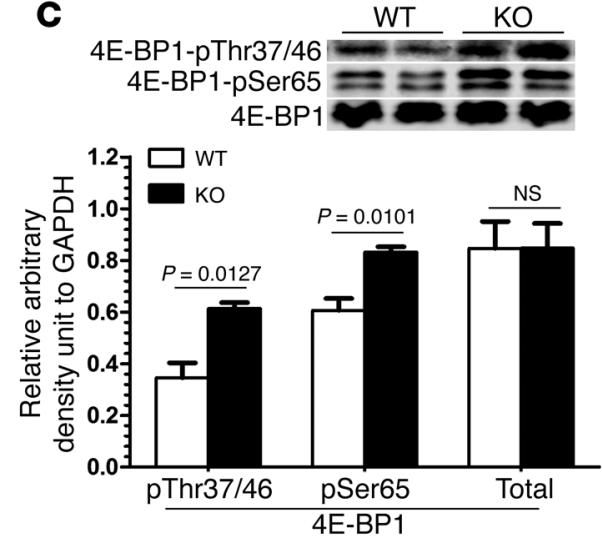

D
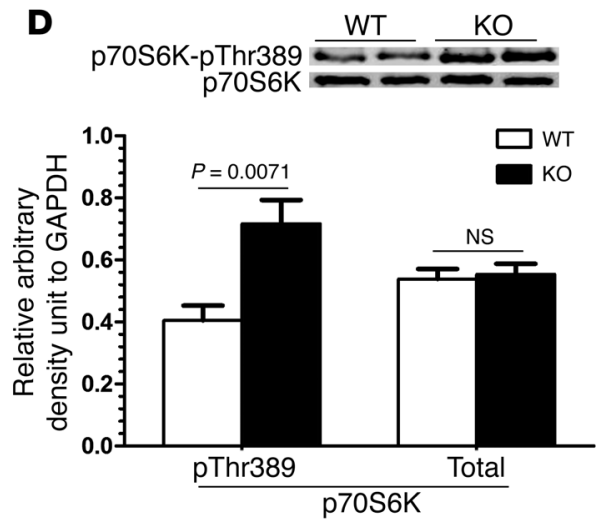

E
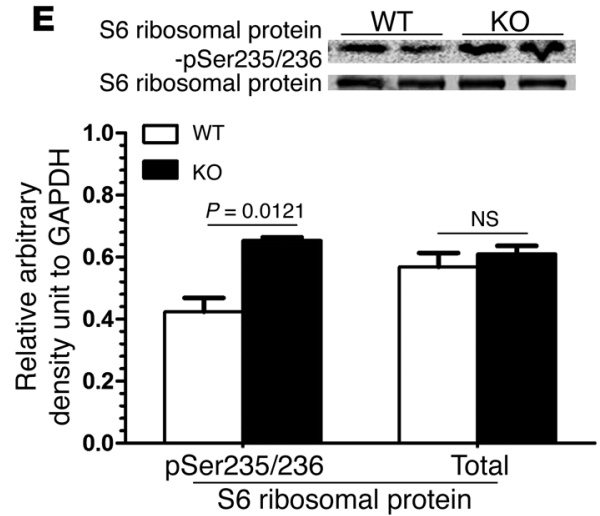

Figure 7

Dysregulation of signaling pathways in the hearts of the Gsk3a KO mice. Immunoblots of 2-year-old WT and Gsk3a KO hearts for the various signaling pathways, including (A) IRS-1; (B) phospho-Akt S473 and Akt; and the 3 mTOR targets (C) 4E-BP1, (D) p70S6 kinase, and (E) S6 ribosomal protein. GAPDH served as an additional loading control in all immunoblots.

mTOR was central to the pathology. Therefore, we treated 1- and 2-year-old Gsk3a KO and WT mice with the mTOR inhibitor, everolimus. Confirming that everolimus was acting as expected to increase autophagy in vitro and in vivo, we found that everolimus pretreatment corrected the defect in starvation-induced autophagic flux seen in the Gsk3a KO fibroblasts (Figure 9A). Everolimus also restored autophagy in MEFs in the presence of the GSK-3 inhibitor SB216763 (Supplemental Figure 4). Taken together, these findings confirm that unrestrained mTOR activation following inhibition or deletion of GSK-3 $\alpha$ is largely responsible for the impaired autophagy that we observed. We also immunoblotted for p62 and LC3-II/I (Figure 9B) and found that everolimus restored p62 and LC3-II/I levels to normal in the KO hearts, consistent with restoration of autophagy. We then asked whether everolimus might reverse the progression of disease seen in the older $\mathrm{KO}$ mice. Everolimus was administered via gavage over 6 weeks, with the mice undergoing periodic transthoracic echocardiography. To our surprise, we saw significant improvement in all functional and morphometric parameters, particularly in the older mice (Figure 10A). The benefit was also seen in the skeletal muscle of the KO mice, as evidenced by a significantly reduced number of skeletal muscle myocytes with vacuolar degeneration (Figure 10B).

In summary, GSK-3 $\alpha$ negatively regulates $\mathrm{mTOR}$ and that inhibition activates autophagy in vitro and appears to do so in vivo. With inhibition or deletion of GSK-3 $\alpha$, mTOR is unrestrained and autophagy is impaired, there is excess accumulation of cellular debris in the striated muscle, and, ultimately, contractile function is reduced. Reestablishing mTOR inhibition downstream of GSK-3 by everolimus restores autophagy as well as contractile function, particularly in the setting of advanced age.

\section{Discussion}

Herein, we present evidence demonstrating that GSK-3 $\alpha$ is a suppressor of aging that retards age-related pathologies, thereby increasing life span in the mouse. Although we focused more on organs with striated muscle, other organ systems were affected as well, including the gut, liver, and bone and joints. In fact, with the exception of skin, which had no obvious aging-related pathologies, every system we examined had significant abnormalities.

Although little has been reported regarding GSK-3s in aging, cues can be found in published studies that imply that GSK-3s have a potential role. For example, GSK-3s are key negative regulators of WNT signaling. Increased WNT signaling may accelerate aging through stimulating protein translation and mitochondrial biogenesis and inducing ROS generation. But in contrast to those findings, we have not observed significant derangements in WNT signaling in the hearts of the Gsk3a KO mice (refs. 29, 40, and data not shown), suggesting that WNT signaling is likely not a major factor in the accelerated aging in the KO heart. We did observe significant increases in ROS in the heart and skeletal 


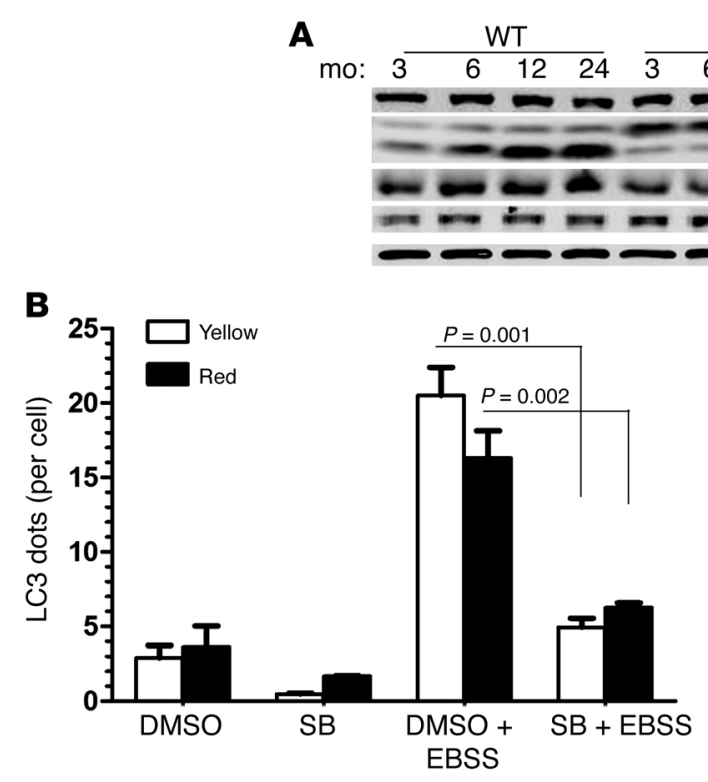

KO
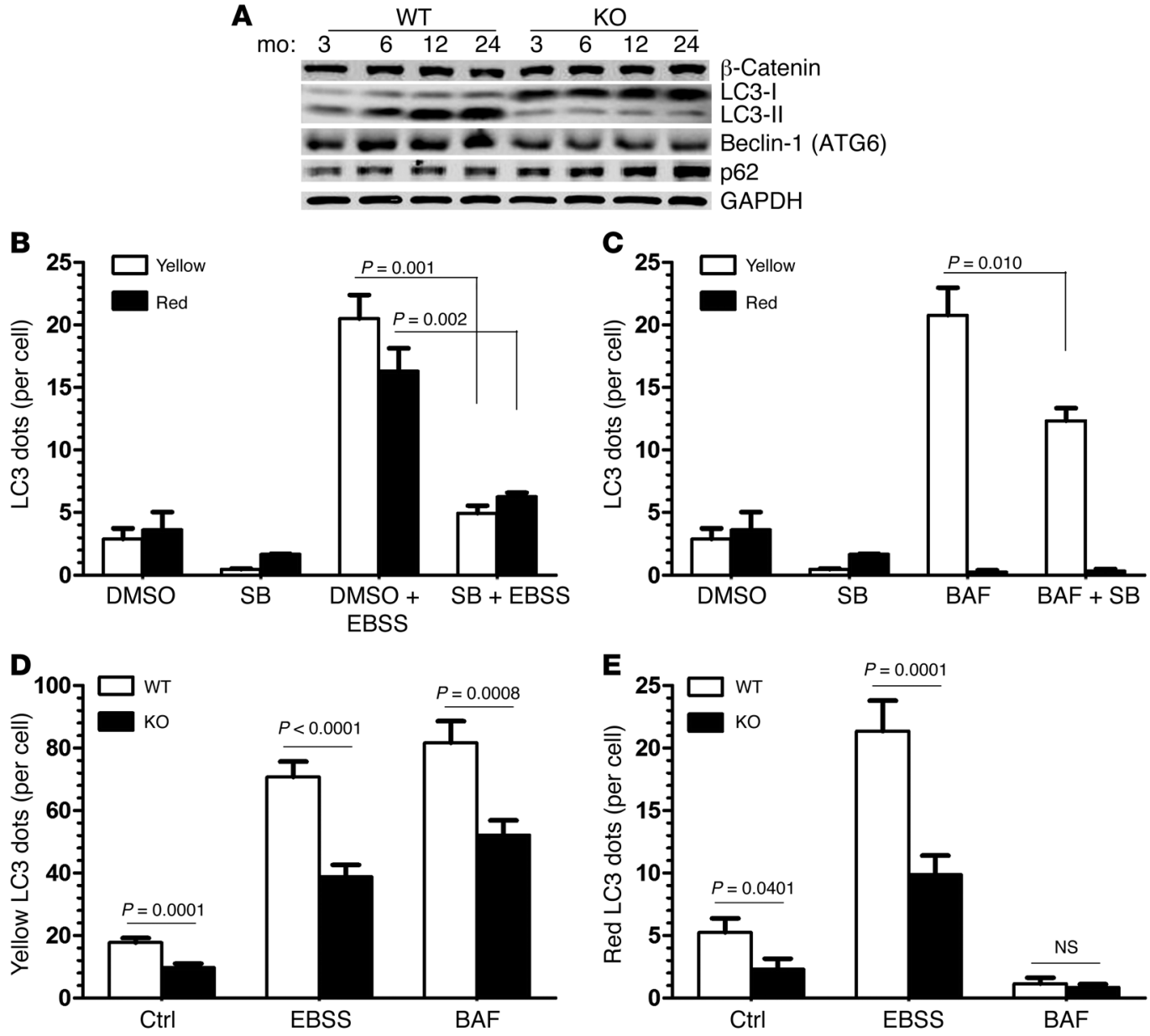

\section{Figure 8}

Impaired autophagy in the hearts of the Gsk3a KO mice. (A) Immunoblot for LC3-I and LC3-II levels in WT and KO mice. Note the markedly lower LC3-II levels in the KO mice compared with those in the WT mice and the higher levels of LC3-I in the KO mice compared with those in the WT mice, particularly evident in the 12- and 24-month-old mice. Beclin-1 (ATG6) levels are decreased whereas p62 levels are increased in the $\mathrm{KO}$ mice, particularly at the 12 and 24 month time points. Immunoblots are consistent with impaired autophagy in the KO mice. (B) Inhibition of autophagy in MEFs treated with a GSK-3 inhibitor (SB216763 [SB]). MEFs were transfected with the tandem mRFP-GFP-LC3 adenovirus. Cells were starved in EBSS buffer in the presence or absence of SB216763, and then the number of yellow and red LC3 dots per cell was quantified by immunofluorescence microscopy. (C) MEFs treated as in D were subjected to the lysosome inhibitor (bafilomycin-A1 [BAF]), the GSK-3 inhibitor SB216763, or both, and LC3 dots were quantified. (D and E) Quantification of yellow and red LC3 dots per cell from the experiment described in $\mathbf{B}$ and $\mathbf{C}$. More than 50 cells were counted in each condition, and data are representative of 3 independent experiments.

muscle of the KO mouse, and this could promote senescence. That said, it is not clear how deletion of GSK-3 $\alpha$ might lead to increased ROS production, and determining the mechanism is beyond the scope of this work.

We do, however, have mechanistic data on dysregulation of 2 key pathways, both of which importantly impinge upon autophagy. Inactivating mutations in IRS proteins, central components of the insulin/IGF-1 signaling pathway, extend life span in various species. IRS-1 has been reported to be phosphorylated by GSK-3 $\beta$, leading to its ubiquitination and proteasomal degradation (41), and, indeed, we saw a significant increase in IRS-1 expression in the heart of the Gsk3a KO mouse. However, this did not appear to lead to enhanced activity of key factors downstream in the IRS-1 pathway, including Akt. Thus, activation of Akt does not appear to be a major mechanism by which autophagy is impaired in the $\mathrm{KO}$ mouse.
However, a second mechanism, and one that we show to be critical to the aging phenotypes, is via the loss of direct regulation of mTORC1 by GSK-3 $\alpha$ in the KO mouse. Inhibiting the mTOR pathway has been shown to increase life span and slow aging-related pathologies. GSK-3, acting via TSC2, leads to inhibition of mTORC1 (42). Our published data have confirmed increased mTORC1 activity in the young ( 4-month-old) Gsk3a KO mouse (31), and this disparity between WT and KO mice is exaggerated with advancing age (43). This unrestrained activation of mTORC1 leads to a profound inhibition of autophagy (Figure 8 and refs. $44,45)$. Each of the 3 markers of autophagy that we examined, beclin-1 (ATG6), LC3-I/II, and p62, were markedly dysregulated, and all indicate impaired autophagy. Beclin-1 is required for the initiation of the formation of the autophagosome, but it was virtually absent in our immunohistochemistry studies. LC3, the mammalian homolog of yeast ATG8, is both a marker and an 
A
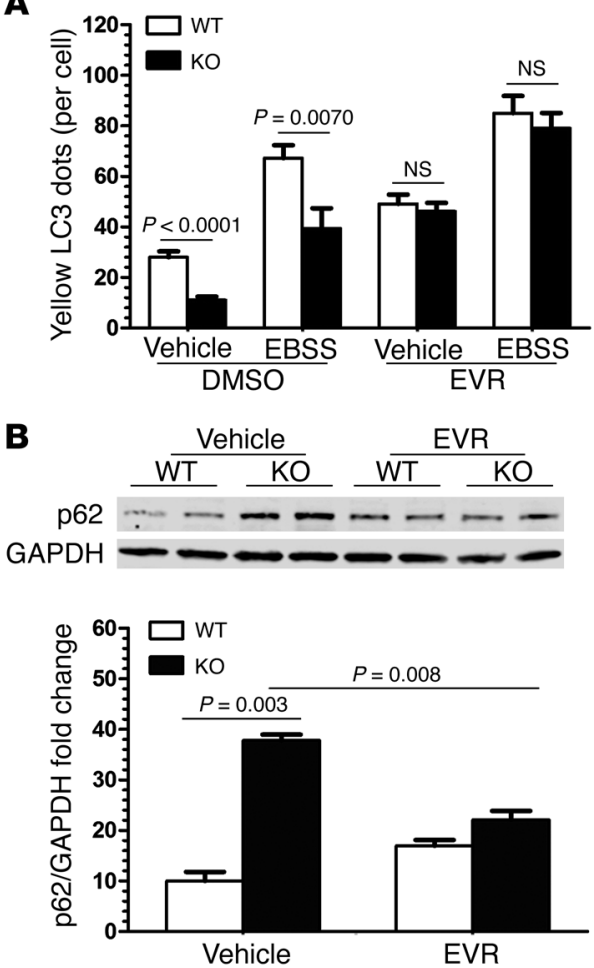
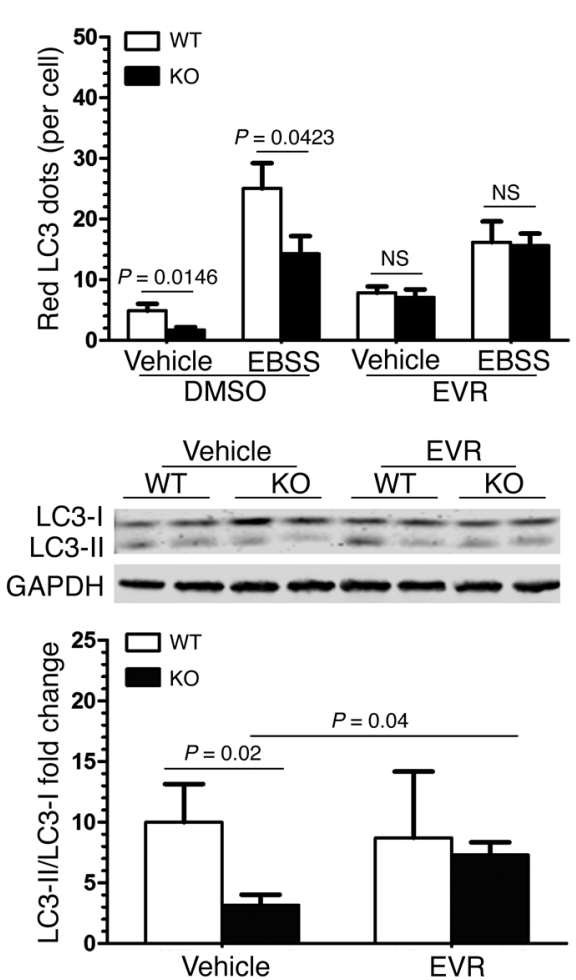

\section{Figure 9}

Inhibition of mTOR restores autophagy in striated muscle of the Gsk3a KO mouse. (A) Adult cardiac fibroblasts were transfected with the tandem mRFP-GFP-LC3 adenovirus. Cells were starved in EBSS buffer, with or without the MTOR inhibitor, everolimus (EVR). The number of yellow and red LC3 dots per cell in each condition was quantified. Data are representative of 3 independent experiments. (B) Two-year-old WT and Gsk3a KO mice were treated with everolimus, as described in the Methods. LV lysates were prepared and autophagy markers were analyzed by Western blot. Densitometric analysis of p62 expression and the LC3-II/LC3-I ratio was normalized to GAPDH. mTOR inhibition largely normalized the impaired autophagy in the aged Gsk3a KO mice. effector of autophagy. When autophagy is blocked, LC3-I levels increase and LC3-II levels decline, and this was strikingly observed in the case of the $\mathrm{KO}$ mice, compared with the WT mice, particularly as the KO mice aged. Finally, p62 is degraded by autophagy, and its increase in expression in the KO mice, particularly with advancing age, is also consistent with impaired autophagy. When viewed in the context of our findings in both skeletal muscle and the heart, which demonstrate an inability of the $\mathrm{KO}$ mice to clear damaged and dysfunctional mitochondria and other debris, we believe impaired autophagy is a key mechanism promoting aging in the $\mathrm{KO}$ mice. In fact, we could not find examples in the literature of such marked dysregulation of these autophagy markers, except in those scenarios that used manipulation of factors directly regulating autophagy. Although autophagy must be viewed as a flux event (and we indeed found striking alterations in autophagic flux following deletion or inhibition of GSK-3 $\alpha$ ), our findings, and those involving the mTOR inhibitor, everolimus (see below), strongly support dysregulation of autophagy as the central driver of the cardiac and skeletal muscle pathologies. Of note, autophagy has also been reported to play an important role in maintaining skeletal muscle mass (46). The pathologies presented in that study, which used skeletal muscle-specific deletion of the autophagy gene, Atg7, are reminiscent of those seen in our studies in both heart and skeletal muscle.

In any case, our studies clearly suggest that increased mTOR activation following deletion of GSK-3 $\alpha$ is the primary mechanism, and final common pathway, summating multiple inputs that lead to impaired autophagy and profound derangements in various tissues. This conclusion is most strongly supported by the studies in which the mTOR inhibitor, everolimus, protected against progression of age-related pathologies in heart and skeletal muscle of younger mice and strikingly reversed those age-re- lated pathologies in older mice This clearly suggests that while the IRS-1/Akt pathway is dysregulated in the Gsk3a KO mouse, its role in the cardiac and skeletal muscle phenotypes is minor. Supporting this conclusion, we found no increase in phosphorylation of T1462, the Akt phosphorylation site on TSC2.

Recently, Lin et al. (47) reported that GSK-3 can, under certain circumstances (i.e., growth factor withdrawal), regulate autophagy, findings that appear to be consistent with our conclusions. However, in contrast to gene deletion, Lin et al. used nonselective small molecule inhibitors and $\mathrm{LiCl}$ to inhibit GSK-3. This limits any firm conclusions from being drawn concerning the role of GSK-3 in general and abrogates the ability to parse out specific roles of the 2 GSK-3 isoforms, since there are no isoform-specific inhibitors. Furthermore, the signaling pathway leading to activation of autophagy appears to be different, since we saw no involvement of the protein TIP60 or AMPK (data not shown). Most importantly, the pathological consequences of alterations in GSK-3 activity and autophagy for multicellular organisms, including regulation of aging, were not addressed in Lin et al.

In conclusion, we believe that our studies define a novel and key role for GSK-3 $\alpha$ in preventing premature aging in several organ systems. In its absence, mTOR is constitutively hyperactivated, and this is associated with derangements in autophagy that have critical consequences on clearing cellular debris and on organismal viability. Our studies open the possibility of moderating the devastating effects of aging by manipulating GSK-3 $\alpha$.

\section{Methods}

The creation of the Gsk3a-/- KO mouse was previously described (48).

Antibodies and chemicals. Antibodies used were directed against $\beta$-catenin (no. 9562), GSK-3 $\alpha$ (no. 9338), GSK-3 $\beta$ (no. 9315), and both phosphorylated GSK-3 $\alpha$ at Ser21 and GSK-3 $\beta$ at Ser9 (no. 9331; all from 
A
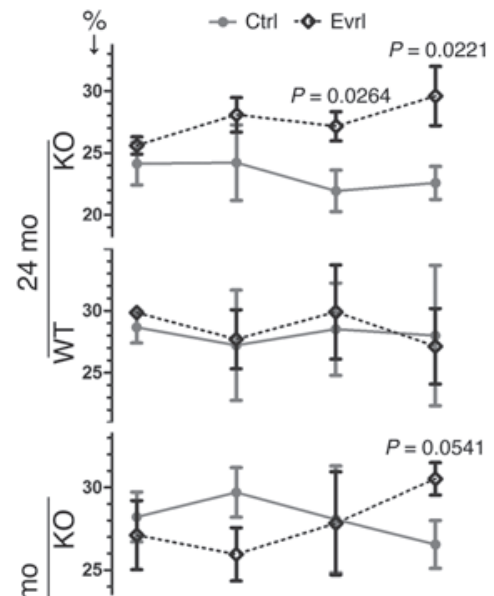

N

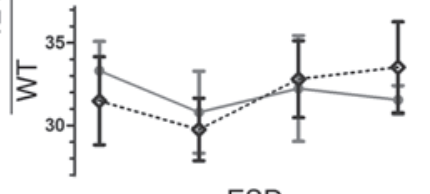

ESD
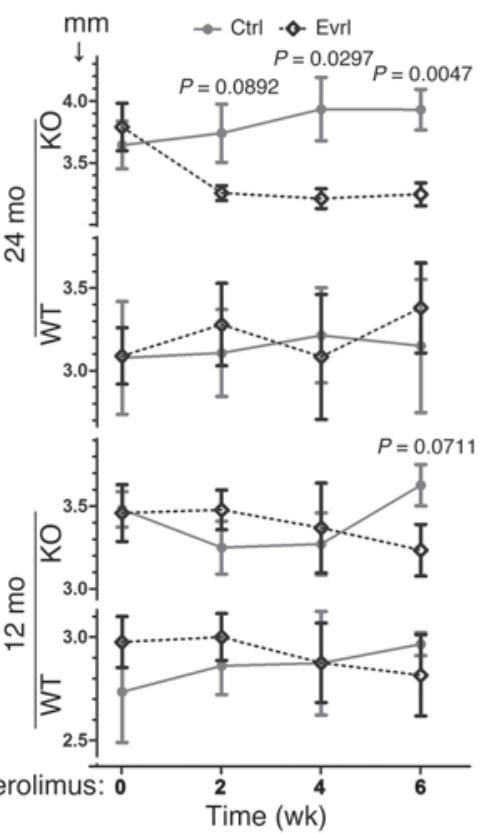

$E F$

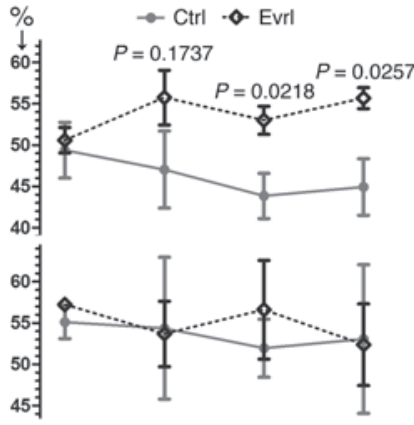

LVPW;
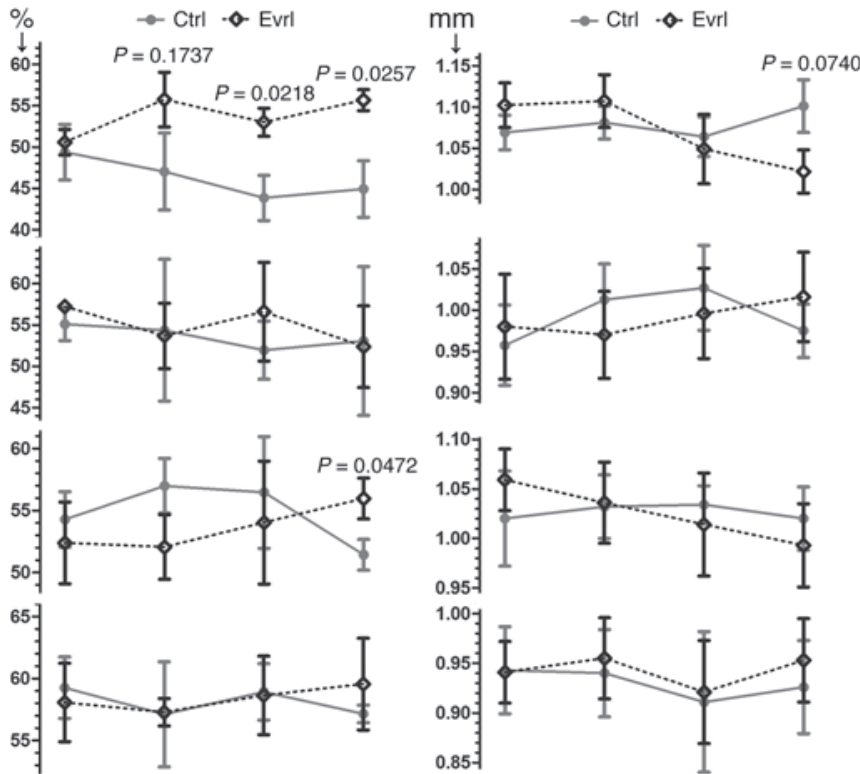

EDD
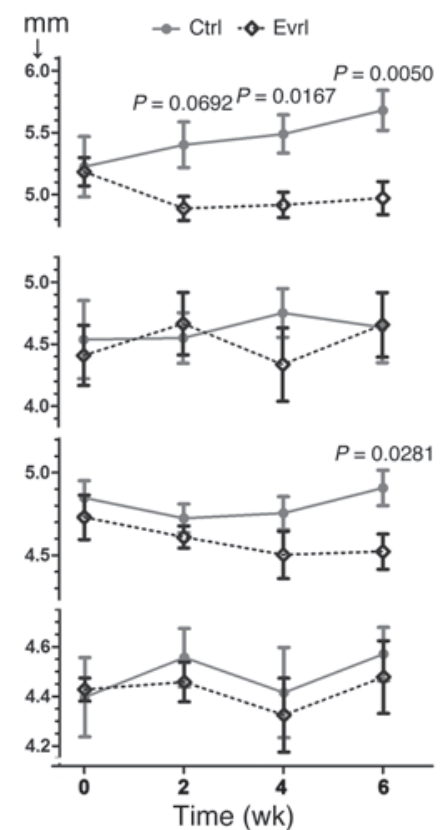

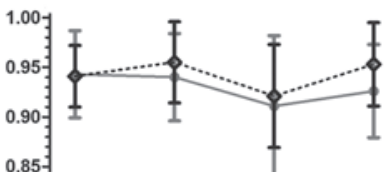

LV mass
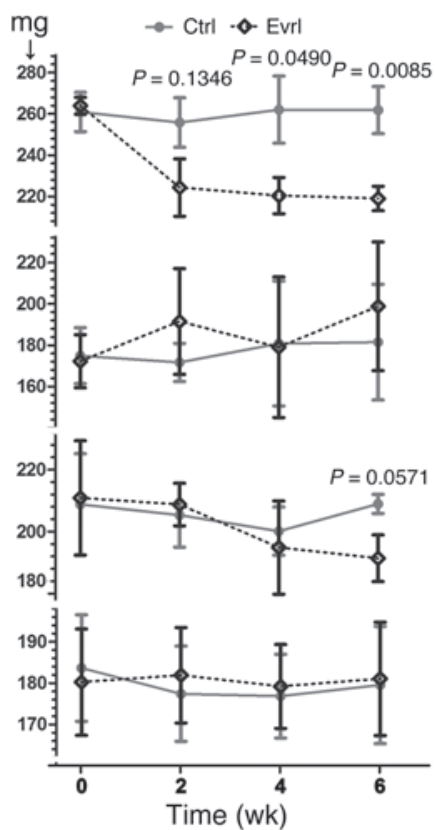
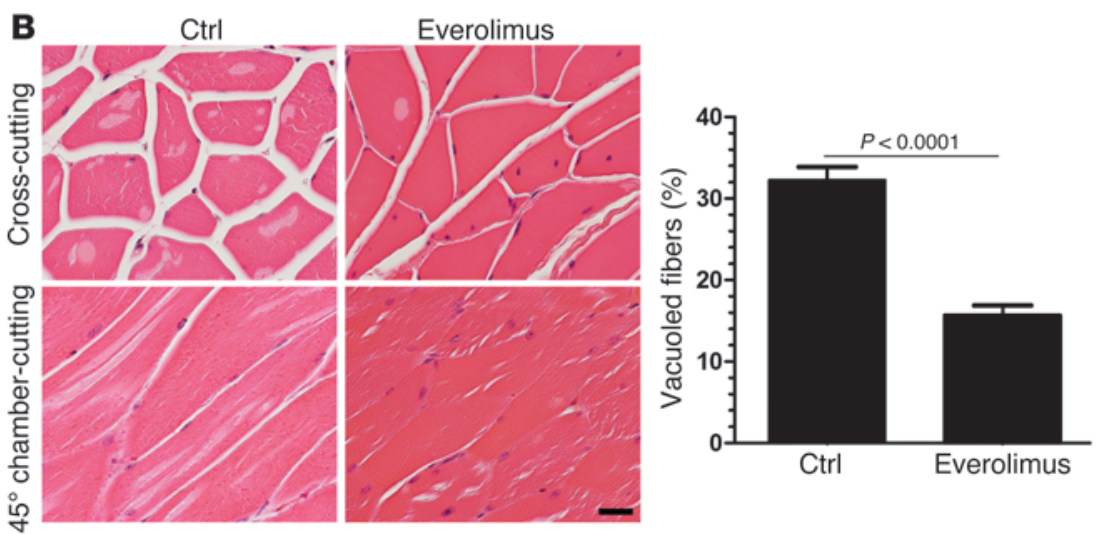

Figure 10

Inhibition of mTOR rescues the aging phenotype in striated muscle of the Gsk3a KO mouse. (A) Contractile function (as determined by FS and EF), hypertrophy (LVPW;d and LV mass), and chamber sizes (ESD and EDD) in Gsk3a WT and KO mice treated with everolimus or placebo. (B) Quantification of vacuolization in skeletal muscle of aging mice treated with everolimus or placebo. Original magnification, $\times 400$; scale bar: $50 \mu \mathrm{m}$. 
Cell Signaling). IRS-1 (no. sc-559) and Beclin-1/ATG6 (no. sc-48381) were from Santa Cruz Biotechnology. H2AX phosphorylated at Ser139 (no. 07-164) was from Millipore. LC3 (no. PD014) was from MBL International. p62 (no. 03-GP62-C) was from ARP Inc.

$\beta$-Galactosidase staining. Cryostat tissue sections were air dried for $25 \mathrm{~min}$ utes at room temperature. Sections were fixed with $0.2 \%$ glutaraldehyde, $5 \mathrm{mM}$ EGTA, and $2 \mathrm{mM} \mathrm{MgCl}_{2}$ in $0.1 \mathrm{M} \mathrm{PB}$ for 10 minutes at $4^{\circ} \mathrm{C}$. Sections were then washed with PBS, twice for 5 minutes each time, and then were rinsed in Detergent Rinse Buffer $\left(2 \mathrm{mM} \mathrm{MgCl}_{2}, 0.02 \%\right.$ Nonidet P- 40 , and $0.01 \%$ sodium deoxycholate in $0.1 \mathrm{M}$ PBS) for 10 minutes. Sections were incubated in X-gal Reaction Buffer $(5 \mathrm{mM}$ potassium ferrocyanide, $5 \mathrm{mM}$ potassium ferricyanide, $2 \mathrm{mM} \mathrm{MgCl} 2,0.02 \%$ Nonidet P- $40,0.01 \%$ sodium deoxycholate, and $1 \mathrm{mg} / \mathrm{ml} \mathrm{X}$-gal in $20 \mathrm{mM}$ Tris buffer, $\mathrm{pH}$ 6.0) overnight at $37^{\circ} \mathrm{C}$ and then washed with PBS, twice for 5 minutes each time. Sections were then placed in $10 \%$ formalin or $4 \%$ paraformaldehyde for 10 minutes at room temperature. They were then washed with PBS, 3 times for 5 minutes each time, counterstained with Nuclear Fast Red (no. H-3403 from Vector Laboratories Inc.) for 3 minutes, washed with PBS twice for 2 minutes each time, then dehydrated with serial concentrations of ethanol (95\%, 3 minutes; $100 \%$, twice for 3 minutes each time), and cleared with xylene twice for 3 minutes each time. Slides were then mounted with permanent mounting media.

Immunohistochemistry. Paraffin sections were deparaffinized with serial xylene washes and rehydrated with serial concentrations of ethanol (from high to low concentrations gradually). To retrieve the antigen, slides were put in Antigen Unmasking Solution (no. H-3300 from Vector Laboratories Inc.) containing $0.1 \%$ Nodidet $\mathrm{P} 40$ for permeabilization. The solution was boiled for 10 minutes in a microwave according to the manufacturer's instructions, and slides were then allowed to cool. Slides were washed with PBS twice for 5 minutes each time and then incubated in $0.3 \%$ hydrogen peroxide in $\mathrm{dd}_{2} \mathrm{O}$ containing $0.2 \%(\mathrm{w} / \mathrm{v})$ sodium azide at room temperature for 10 minutes to eliminate activity of endogenous peroxidases. Sections were incubated in blocking buffer $(5 \%[\mathrm{v} / \mathrm{v}]$ normal serum in PBS) for 30 minutes at room temperature. Sections were incubated with primary antibody at $1: 250$ in blocking buffer at $4^{\circ} \mathrm{C}$ overnight. Slides were washed with PBS 3 times for 5 minutes each time and then incubated with the EnVision+ System-HRP (no. K4002 from Dako) for 60 minutes at room temperature, followed by washing with PBS, 3 times for 5 minutes each time, and development with DAB substrate from the Peroxidase Substrate Kit (no. SK-4100 from Vector Laboratories Inc.). Slides were counterstained with Hematoxylin QS (no. H-3404 from Vector Laboratories Inc.) and then were dehydrated with serial concentrations of ethanol and cleared with serial xylene washes. Slides were mounted with permanent mounting media.

Immunoblotting. LV tissue was homogenized in 10 volumes of lysis buffer (50 mM Tris-HCl, pH 7.4, 150 mM NaCl, 1 mM EDTA, 0.25\% sodium deoxycholate, $1 \% \mathrm{NP}-40$ ), supplemented with protease inhibitor cocktail and phosphatase inhibitor cocktail (Sigma-Aldrich). After homogenization, the homogenates were centrifuged at $12,000 \mathrm{~g}$ for 15 minutes and separated into NP-40 soluble supernatant and insoluble pellet. Protein concentration in the supernatant was quantified with the bicinchoninic acid protein assay (no. 23225 from Pierce). The supernatant was loaded for immunoblotting unless otherwise noted. Equal amounts of proteins were subjected to SDS-PAGE and subsequently were transferred to nitrocellulose membranes. Primary antibody incubations were performed at 1:1,000 dilution. All incubations were done at $4^{\circ} \mathrm{C}$, overnight. The secondary antibody used was Alexa Fluor 680 (Molecular Probes), at 1:2,500 dilution, for 1 hour at room temperature. Membranes were scanned with the Odyssey Infrared Imaging System (LI-COR).

Detection of superoxide production. Superoxide production in cardiac and skeletal muscle was measured by lucigenin-enhanced chemiluminescence, as previously described (49). In brief, tissue homogenates were placed in lucigenin buffer ( $5 \mu \mathrm{mol} / \mathrm{l})$, and relative light units (RLUs) were measured with an FB 12 luminometer. Superoxide production was expressed as RLUs per second per mg wet tissue.

Echocardiography. Transthoracic 2-dimensional echocardiography was performed with a $12-\mathrm{mHz}$ probe (VisualSonics) on mice anesthetized by inhalation of isoflurane ( $1 \% \sim 1.5 \%)$. M-mode interrogation was performed in the parasternal short-axis view at the level of the greatest LV end-diastolic dimension (EDD). EDD, LV end-systolic dimension (ESD), and diastolic LV posterior wall thickness (LVPW; d) were measured and used to calculate percentage of fractional shortening (FS), EF, and LV mass. FS and EF values were exported from the echo program, and LV mass was calculated with the following formula: $(2 \times \text { LVPW; }+\mathrm{EDD})^{3}-\mathrm{EDD}^{3}$.

Hemodynamics. For in vivo hemodynamic measurements, a 1.4 French micromanometer-tipped catheter (no. SPR-671 from Millar Instruments Inc.) was inserted into the right carotid artery and advanced into the $\mathrm{LV}$ of mice that were lightly anesthetized (i.e., maintained spontaneous respirations) with tribromoethanol/amylene hydrate $(2.5 \% \mathrm{w} / \mathrm{v}, 8 \mu \mathrm{l} / \mathrm{g}$ injected intraperitoneal; Avertin). Hemodynamic parameters, including LV systolic pressure, $\mathrm{LV}$ end-diastolic pressure, and rate of $\mathrm{LV}$ pressure rise $(+\mathrm{dP} / \mathrm{dt}$ and $-\mathrm{dP} / \mathrm{dt}$ ), were recorded in closed-chest mode, both at baseline and in response to $10 \mathrm{ng}$ isoproterenol, administered via cannulation of the right internal jugular vein.

Micro-CT analysis. The knee joint was analyzed by micro-CT, as previously described (50). Briefly, 6 right knee joints were harvested from $\mathrm{KO}$ mice and littermate controls, were fixed in $4 \%$ paraformaldehyde, and then subjected to micro-CT analysis (Scanco $\mu \mathrm{CT} 40$ ).

Autophagic flux. MEF cells were maintained in DMEM with 10\% FBS supplemented with L-glutamine, and penicillin/streptomycin. Adult cardiac fibroblasts were isolated from WT and Gsk3a KO mice, as previously described (51). The method to evaluate tandem fluorescent LC3 puncta using Ad-mRFP-LC3 has been described previously (52). Briefly, MEF cells were transfected with Ad-mRFP-LC3 at 100 MOI for 24 hours (a gift from J. Sadoshima, University of Medicine and Dentistry of New Jersey, Newark, New Jersey, USA). For, starvation, cells were first washed with PBS 3 times and then incubated in EBSS (SH30029, Hyclone) for 4 hours. To inhibit autophagosome-lysosome fusion, MEFs were treated with $50 \mathrm{nmol} / \mathrm{l}$ bafilomycin-A1 for 4 hours. After designated treatments, cells were washed twice with PBS and fixed with $4 \%$ paraformaldehyde in PBS. All the cellular images were obtained using a Nikon TiE fluorescence microscope. For quantification of autophagic cells, GFP-LC3 and mRFP-LC3 punctated dots were determined from triplicates by manual counting of more than 50 cells. In this assay, mRFP retains its fluorescence, even in the acidic environment of lysosomes, whereas GFP loses its fluorescence.

Statistics. Differences between data groups were evaluated for significance using unpaired 2-tailed Student's $t$ test or 1-way ANOVA, as appropriate, and Bonferroni post-hoc test (GraphPad Prism Software Inc.). Repeated -measures ANOVA was used to evaluate the statistical significance of data acquired from same animals over multiple time points. Survival analysis was performed by the Kaplan-Meier method, and between-group differences in survival were tested by the Gehan-Breslow-Wilcoxon test. Data are expressed as mean $\pm \mathrm{SEM}$, unless noted otherwise. For all tests, $P<0.05$ was considered statistically significant.

Study approval. All studies involving the use of animals were approved by the IACUC of the Temple University School of Medicine.

\section{Acknowledgments}

This work was supported by grants from the NHLBI to T. Force, a CIHR operating grant (FRN 12858) to J. Woodgett, an NIDCR grant (R03 DE020840-03) to T. Freeman, and American Diabetes 
Association Junior Faculty Award (1-11-JF-56) to Y. Wang. We acknowledge Brian Moore and Christine Macolino for help with bone histology and micro-CT.

Received for publication May 3, 2012, and accepted in revised form January 24, 2013.

1. Kirkwood TB, Austad SN. Why do we age? Nature. 2000;408(6809):233-238.

2. von Figura G, Hartmann D, Song Z, Rudolph $\mathrm{KL}$. Role of telomere dysfunction in aging and its detection by biomarkers. J Mol Med. 2009; 87(12):1165-1171.

3. Wang C, Jurk D, Maddick M, Nelson G, Martin-Ruiz C, von Zglinicki T. DNA damage response and cellular senescence in tissues of aging mice. Aging Cell. 2009;8(3):311-323.

4. Sahin E, et al. Telomere dysfunction induces metabolic and mitochondrial compromise. Nature. 2011; 470(7334):359-365.

5. Hoeijmakers JH. DNA damage, aging, and cancer. NEngl J Med. 2009;361(15):1475-1485.

6. Vellai T, Takacs-Vellai K, Sass M, Klionsky DJ. The regulation of aging: does autophagy underlie longevity? Trends Cell Biol. 2009;19(10):487-494.

7. Madeo F, Tavernarakis N, Kroemer G. Can autophagy promote longevity? Nat Cell Biol. 2010; 12(9):842-846

8. Partridge L, Piper MD, Mair W. Dietary restriction in Drosophila. Mech Ageing Dev. 2005;126(9):938-950.

9. Kuningas M, Mooijaart SP, van Heemst D, Zwaan BJ, Slagboom PE, Westendorp RG. Genes encoding longevity: from model organisms to humans. Aging Cell. 2008;7(2):270-280.

10. Apfeld J, Kenyon C. Cell nonautonomy of C. elegans daf- 2 function in the regulation of diapause and life span. Cell. 1998;95(2):199-210.

11. Holzenberger $M$, et al. IGF-1 receptor regulates lifespan and resistance to oxidative stress in mice. Nature. 2003;421(6919):182-187.

12. Bluher M, Kahn BB, Kahn CR. Extended longevity in mice lacking the insulin receptor in adipose tissue. Science. 2003;299(5606):572-574.

13. Foster KG, Fingar DC. Mammalian target of rapamycin (mTOR): conducting the cellular signaling symphony. J Biol Chem. 2010;285(19):14071-14077.

14. Gingras AC, Raught B, Sonenberg N. mTOR signaling to translation. Curr Top Microbiol Immunol. 2004; 279:169-197.

15. Selman C, et al. Ribosomal protein S6 kinase 1 signaling regulates mammalian life span. Science. 2009; 326(5949):140-144.

16. Hands SL, Proud CG, Wyttenbach A. mTOR's role in ageing: protein synthesis or autophagy? Aging (Albany NY). 2009;1(7):586-597.

17. Yoon JC, Ng A, Kim BH, Bianco A, Xavier RJ, Elledge SJ. Wnt signaling regulates mitochondrial physiology and insulin sensitivity. Genes Dev. 2010; 24(14):1507-1518.

18. Vousden KH, Prives C. Blinded by the light: the growing complexity of p53. Cell. 2009;137(3):413-431.

19. Budanov AV, Karin M. p53 target genes sestrin 1 and sestrin 2 connect genotoxic stress and mTOR

Address correspondence to: Thomas Force, Professor of Medicine, Temple University School of Medicine, Clinical Director, Center for Translational Medicine, Temple University School of Medicine, 3500 N. Broad St., MERB 943, Philadelphia, Pennsylvania 19140, USA. Phone: 215.707.9829; Fax: 215.707.9890; E-mail: thomas. force@temple.edu.

signaling. Cell. 2008;134(3):451-460

20. Budanov AV, Lee JH, Karin M. Stressin' Sestrins take an aging fight. EMBO Mol Med. 2010; 2(10):388-400.

21. Lee JH, Bodmer R, Bier E, Karin M. Sestrins at the crossroad between stress and aging. Aging (Albany NY). 2010;2(6):369-374.

22. Kuro-o M, et al. Mutation of the mouse klotho gene leads to a syndrome resembling ageing. Nature. 1997;390(6655):45-51.

23. Liu H, et al. Augmented Wnt signaling in a mammalian model of accelerated aging. Science. 2007; 317(5839):803-806

24. Woodgett JR, Tonks NK, Cohen P. Identification of a calmodulin-dependent glycogen synthase kinase in rabbit skeletal muscle, distinct from phosphorylase kinase. FEBS Lett. 1982;148(1):5-11.

25. Dorn GW 2nd, Force T. Protein kinase cascades in the regulation of cardiac hypertrophy. J Clin Invest. 2005;115(3):527-537.

26. Doble BW, Woodgett JR. GSK-3: tricks of the trade for a multi-tasking kinase. J Cell Sci. 2003; 116(pt 7):1175-1186.

27. Force T, Woodgett JR. Unique and overlapping functions of GSK-3 isoforms in cell differentiation and proliferation and cardiovascular development. J Biol Chem. 2009;284(15):9643-9647.

28. Xu C, Kim NG, Gumbiner BM. Regulation of protein stability by GSK3 mediated phosphorylation. Cell Cycle. 2009;8(24):4032-4039.

29. Kaidanovich-Beilin O, Woodgett JR. GSK-3: functional insights from cell biology and animal models. Front Mol Neurosci. 2011;4:40.

30. Cheng H, Woodgett J, Maamari M, Force T. Targeting GSK-3 family members in the heart: a very sharp double-edged sword. J Mol Cell Cardiol. 2011; 51(4):607-613.

31. Zhou J, et al. GSK-3alpha directly regulates beta-adrenergic signaling and the response of the heart to hemodynamic stress in mice. J Clin Invest. 2010; 120(7):2280-2291.

32. Schiaffino S. Tubular aggregates in skeletal muscle: Just a special type of protein aggregates? Neuromuscul Disord. 2012;22(3):199-207.

33. Pavlovicova M, Novotova M, Zahradnik I. Structure and composition of tubular aggregates of skeletal muscle fibres. Gen Physiol Biophys. 2003;22(4):425-440.

34. MacAulay K, et al. Glycogen synthase kinase 3 alpha-specific regulation of murine hepatic glycogen metabolism. Cell Metab. 2007;6(4):329-337.

35. Inoki K, Li Y, Zhu T, Wu J, Guan KL. TSC2 is phosphorylated and inhibited by Akt and suppresses mTOR signalling. Nat Cell Biol. 2002;4(9):648-657.

36. Barth S, Glick D, Macleod KF. Autophagy: assays and artifacts. J Pathol. 2010;221(2):117-124.

37. Glick D, Barth S, Macleod KF. Autophagy: cel- lular and molecular mechanisms. J Pathol. 2010; 221(1):3-12.

38. Mizushima N, Yoshimori T, Levine B. Methods in mammalian autophagy research. Cell. 2010; 140(3):313-326

39. Hariharan N, Zhai P, Sadoshima J. Oxidative stress stimulates autophagic flux during ischemia/reperfusion. Antioxid Redox Signal. 2011;14(11):2179-2190.

40. Kerkela R, et al. Deletion of GSK-3beta in mice leads to hypertrophic cardiomyopathy secondary to cardiomyoblast hyperproliferation. J Clin Invest. 2008; 118(11):3609-3618.

41. Leng S, et al. Glycogen synthase kinase 3 beta mediates high glucose-induced ubiquitination and proteasome degradation of insulin receptor substrate 1 . J Endocrinol. 2010;206(2):171-181.

42. Inoki $\mathrm{K}$, et al. TSC2 integrates Wnt and energy signals via a coordinated phosphorylation by AMPK and GSK3 to regulate cell growth. Cell. 2006; 126(5):955-968.

43. Jin T, George Fantus I, Sun J. Wnt and beyond Wnt: multiple mechanisms control the transcriptional property of beta-catenin. Cell Signal. 2008; 20(10):1697-1704.

44. Levine B, Kroemer G. Autophagy in the pathogenesis of disease. Cell. 2008;132(1):27-42.

45. Kroemer G, Marino G, Levine B. Autophagy and the integrated stress response. Mol Cell. 2010; 40(2):280-293.

46. Masiero E, et al. Autophagy is required to maintain muscle mass. Cell Metab. 2009;10(6):507-515.

47. Lin SY, et al. GSK3-TIP60-ULK1 signaling pathway links growth factor deprivation to autophagy. Science. 2012;336(6080):477-481.

48. Doble BW, Patel S, Wood GA, Kockeritz LK, Woodgett JR. Functional redundancy of GSK-3alpha and GSK-3beta in Wnt/beta-catenin signaling shown by using an allelic series of embryonic stem cell lines. Dev Cell. 2007;12(6):957-971.

49. Wang Y, et al. AMP-activated protein kinase deficiency enhances myocardial ischemia/reperfusion injury but has minimal effect on the antioxidant/ antinitrative protection of adiponectin. Circulation. 2009;119(6):835-844.

50. Freeman TA, Patel P, Parvizi J, Antoci V Jr, Shapiro IM. Micro-CT analysis with multiple thresholds allows detection of bone formation and resorption during ultrasound-treated fracture healing. J Orthop Res. 2009;27(5):673-679.

51. Lal H, et al. Glycogen synthase kinase-3alpha limits ischemic injury, cardiac rupture, post-myocardial infarction remodeling and death. Circulation. 2012;125(1):65-75

52. Ni HM, et al. Dissecting the dynamic turnover of GFP-LC3 in the autolysosome. Autophagy. 2011; 7(2):188-204 